\title{
Targeting cellular senescence prevents age-related bone loss in mice
}

\author{
Joshua N. Farr, Ph.D. ${ }^{\dagger}$, Ming Xu, Ph.D ${ }^{\dagger}$, Megan M. Weivoda, Ph.D ${ }^{\dagger}$, David G. Monroe, Ph.D., \\ Daniel G. Fraser, Ph.D., Jennifer L. Onken, B.S., Brittany A. Negley, B.S., Jad G. Sfeir, M.D., \\ Mikolaj B. Ogrodnik, B.S., Christine M. Hachfeld, V.T., Nathan K. LeBrasseur, P.T., Ph.D., \\ Matthew T. Drake, M.D., Ph.D., Robert J. Pignolo, M.D., Ph.D., Tamar Pirtskhalava, Ph.D., \\ Tamara Tchkonia, Ph.D., Merry Jo Oursler, Ph.D., James L. Kirkland, M.D., Ph.D. ${ }^{*}$ and \\ Sundeep Khosla, M.D.* \\ Robert and Arlene Kogod Center on Aging and Division of Endocrinology, Mayo Clinic College of \\ Medicine, Rochester, MN, 55905
}

\begin{abstract}
Aging is associated with increased cellular senescence, which is hypothesized to drive the eventual development of multiple co-morbidities ${ }^{1}$. Here, we investigate a role for senescent cells in agerelated bone loss by multiple approaches. In particular, we used either genetic (i.e., the INK$A T T A C$ "suicide" transgene encoding an inducible caspase 8 expressed specifically in senescent cells ${ }^{2-4}$ ) or pharmacological (i.e., "senolytic" compounds ${ }^{5,6}$ ) means to eliminate senescent cells. We also inhibited the production of the pro-inflammatory secretome of senescent cells using a JAK inhibitor (JAKi) ${ }^{3,7}$. In old (20-22-months) mice with established bone loss, activation of the INK-ATTAC caspase 8 in senescent cells or treatment with senolytics or the JAKi for 2-4 months resulted in higher bone mass and strength and better bone microarchitecture compared to vehicletreated mice. The beneficial effects of targeting senescent cells were due to lower bone resorption with either maintained (trabecular bone) or higher (cortical bone) bone formation as compared to vehicle-treated mice. In vitro studies demonstrated that senescent cell-conditioned medium impaired osteoblast mineralization and enhanced osteoclast progenitor survival, leading to increased osteoclastogenesis. Collectively, these data establish a causal role for senescent cells in
\end{abstract}

Users may view, print, copy, and download text and data-mine the content in such documents, for the purposes of academic research, subject always to the full Conditions of use: http://www.nature.com/authors/editorial_policies/license.html\#terms

"Co-corresponding authors: Sundeep Khosla, M.D., College of Medicine, Mayo Clinic, 200 First Street SW, Rochester, Minnesota 55905; Tel: 507-255-6663; khosla.sundeep@ mayo.edu. James L. Kirkland, M.D., Ph.D., College of Medicine, Mayo Clinic, 200 First Street SW, Rochester, Minnesota 55905; Tel: 507-266-9151; kirkland.james@mayo.edu.

$\dagger$ These authors contributed equally to this work.

Supplementary materials: This manuscript contains supplemental materials.

Author Contributions. J.N.F. performed most experiments/analyses on INK-ATTAC and D+Q treated mice. M.X. generated conditioned medium and defined the SASP mechanism. M.M.W. performed osteoclast cell culture experiments. M.X. and M.M.W. performed most experiments/analyses on JAKi treated mice. D.G.M. provided technical guidance. D.G.F., J.L.O., B.A.N., J.G.S., M.B.O., C.M.H., T.P., T.T. N.K.L., M.T.D., R.J.P, and M.J.O assisted with various aspects of the experiments/analyses. J.N.F., M.X., M.M.W., T.T., J.L.K., and S.K. contributed to the design of experiments. J.N.F., M.W., and S.K. wrote the manuscript with input from all co-authors. S.K. directed and supervised all aspects of the study in collaboration with J.L.K. All authors reviewed the manuscript.

Competing Financial Interests: JLK, TT, TP have a financial interest related to this research. A patent on senolytic drugs (WO2015116735A1) is held by Mayo Clinic. This research has been reviewed by the Mayo Clinic Conflict of Interest Review Board and was conducted in compliance with Mayo Clinic Conflict of Interest policies. None of the other authors have a relevant conflict of financial interest. 
bone loss with aging and demonstrate that targeting these cells has both anti-resorptive and anabolic effects on bone. As eliminating senescent cells and/or inhibiting their pro-inflammatory secretome also improves cardiovascular function ${ }^{4}$, enhances insulin sensitivity ${ }^{3}$, and reduces frailty $^{7}$, targeting this fundamental mechanism to prevent age-related bone loss suggests a novel treatment strategy not only for osteoporosis but also for multiple age-related co-morbidities.

\section{Keywords}

Aging; Osteocyte; Osteoclast; Disease prevention; Drug therapy

Accumulation of DNA damage and/or other cellular stressors (e.g., oncogenic insults, reactive metabolites, proteotoxic stress $)^{1,8-10}$ cause proliferating ${ }^{11,12}$ as well as terminally differentiated, non-dividing cells ${ }^{13-16}$ to undergo senescence, characterized by profound chromatin and secretome changes. Cellular senescence is also associated with increased expression of the senescence biomarker, $C d k n 2 a\left(p 16^{I n k 4 a}\right)$, and resistance to apoptosis ${ }^{1,17}$. In addition, senescent cells can develop the senescence-associated secretory phenotype (SASP), consisting of pro-inflammatory cytokines, chemokines, and extracellular matrixdegrading proteins, which have deleterious paracrine and systemic effects ${ }^{3,18-20}$. Indeed, even a relatively low abundance of senescent cells (e.g., $\sim 10-15 \%$ in old primates ${ }^{21}$ ) is sufficient to cause tissue dysfunction. We recently demonstrated that with aging, multiple cell types within the bone microenvironment become senescent, although senescent myeloid cells and senescent osteocytes predominantly develop the $\mathrm{SASP}^{16}$. Consistent with this, further characterization revealed that $p 16^{I n k 4 a}$ expression in mouse osteocytes increases markedly after $\sim 18$ months of age in both sexes (Supplementary Fig. 1a,b), coinciding with the timing of accelerated age-related bone loss in both female and male mice (Supplementary Fig. 1c-j) $)^{22,23}$.

Eliminating a relatively small proportion $(\sim 30 \%)$ of senescent cells using a "suicide" transgene, INK-ATTAC, that permits inducible elimination of $p 16^{\text {Ink } 4 a}$-expressing senescent cells upon administration of a drug (AP20187; Supplementary Fig. 2), extends healthspan and prevents the development of multiple age-related morbidities in both progeroid and normal chronologically-aged mice ${ }^{2-4}$. However, the skeletal phenotype of these animals has not been characterized and the potential role of senescent cells in age-related bone loss has not been investigated. To test the hypothesis that senescent cells mediate age-related bone loss, female INK-ATTAC transgenic mice ${ }^{2-4}$ were randomized to either vehicle or AP20187 treatment twice weekly for 4 months, starting at 20 months of age (Fig. 1a). As anticipated, AP20187 treatment resulted in markedly lower $p 16^{I n k 4 a}$ mRNA expression (by $-59 \%$ ) in bone relative to vehicle-treated mice (Fig. 1b) as well as lower EGFP mRNA (by $-48 \%$ ) encoded by the INK-ATTAC transgene ${ }^{2-4}$ (Fig. 1c), consistent with clearance of senescent cells. This was confirmed by fewer senescent osteocytes in AP20187- versus vehicle-treated mice (by $-46 \%$ ), as assessed by an established senescence biomarker (senescence-associated distension of satellites [SADS $]^{9,16}$ (Fig. 1d-f); see Supplementary Fig. 3 and legend for a further, detailed in vitro validation of the SADS assay using primary osteocyte cultures $)^{9,16}$. Note that we used three measures of senescent cell burden in bone ( $p 16^{I n k 4 a}$ mRNA, EGFP mRNA encoded by the INK-ATTAC transgene, and SADS-positive osteocytes), all with 
concordantly lower values in AP20187- versus vehicle-treated mice. The systemic clearance of senescent cells by AP20187 treatment was further demonstrated by lower $p 16^{\text {Ink4a }}$ (Fig. $1 \mathrm{~g})$ and $E G F P$ (Fig. 1h) mRNA levels in adipose tissue.

Relative to vehicle treatment, AP20187-treated mice had better spine trabecular bone microarchitecture (e.g., 52\% higher BV/TV; Fig. 1i-n), with similar improvements in trabecular bone at the femur (Supplementary Fig. 4a-f). AP20187 treatment also resulted in significantly higher cortical thickness and bone strength (by micro-finite element analysis $[\mu \mathrm{FEA}])$ at the femur (Fig. 1o-q). In addition, consistent with the $\mu$ FEA results, we found significantly better caudal vertebrae bone biomechanical properties (assessed by biomechanical testing ${ }^{24}$ ) in AP20187- as compared to vehicle-treated mice (Supplementary Fig. 5a-e), whereas bone material properties (assessed by nanoindentation testing ${ }^{25,26)}$ ) were not different between AP20187- and vehicle-treated mice (Supplementary Fig. 5f,g).

In contrast to the old mice, treatment of young adult (12-month-old) INK-ATTAC mice with AP20187 did not alter bone parameters (Supplementary Fig. 6a-o), indicating that this strategy is specific for prevention of age-related bone loss. Trabecular bone histomorphometry in the old INK-ATTAC mice demonstrated significantly lower bone resorption (osteoclast numbers per bone perimeter; Supplementary Fig. 7a) in AP20187versus vehicle-treated mice, without a coupled reduction in bone formation indices (osteoblast numbers, mineral apposition rate, and bone formation rate [Supplementary Fig. 7b-d]). We excluded possible direct effects of AP20187 on osteoclasts by demonstrating that whereas treatment for 4 months in old INK-ATTAC mice resulted in significantly lower concentrations of the circulating bone resorption marker, C-terminal telopeptide of type I collagen (CTx), treatment of young INK-ATTAC mice for either 1.5 months or 4 months had no effect on CTx levels (Supplementary Fig. 8a). In addition, in vitro treatment of mature osteoclasts generated from the INK-ATTAC mouse bone marrow with AP20187 did not induce osteoclast apoptosis (Supplementary Fig. 8b-d). Despite these findings, however, it is still possible that with aging, a subset of osteoclasts become senescent and may be directly cleared by AP20187, thereby contributing to the observed inhibition of bone resorption.

As shown in Supplementary Fig. 8e, osteoblast numbers were markedly lower in old as compared to young mice and were unchanged by AP20187 treatment. By contrast, AP20187 treatment resulted in significantly lower osteoclast numbers (Supplementary Fig. 8f), resulting in an improvement in the osteoblast:osteoclast ratio in the old AP20187-treated mice (Supplementary Fig. 8g). The lower bone resorption in AP20187- versus vehicletreated mice was also evident on the endocortical surface of the femur (Fig. 1r); additionally, on this surface, osteoblast numbers, mineral apposition rate, and bone formation rate were all higher in the AP20187- versus vehicle-treated mice (Fig. 1s-u), thus suggesting that the accumulation of senescent cells led to an impairment in bone formation that was improved by their clearance. To further test this, osteoblastic MC3T3 cells were exposed to conditioned medium (CM) collected from control or senescent cells. Senescent cell CM treatment markedly impaired the mineralization of MC3T3 cells as compared to control CM (Fig. 1v,w). Therefore, reduction in senescent cell burden in old (but not young) mice using a genetic approach resulted in improved trabecular and cortical bone parameters associated with lower bone resorption and either maintained (trabecular bone) or higher (cortical bone) 
bone formation. To further define potential mechanisms, we assessed mRNA expression of key regulators of bone formation (Sost) and bone resorption [Tnfrs $11 \mathrm{~b}(\mathrm{Opg})$, and Tnfsf11 $\left(\right.$ Rankl)] in osteocyte-enriched bones ${ }^{16,27}$ from these mice and found significantly lower Sost mRNA expression in AP20187- versus vehicle-treated mice (Supplementary Fig. 9a-d). Clearance of senescent cells also resulted in significantly lower numbers, perimeter, and volume/tissue volume of bone marrow adipocytes (Supplementary Fig. 9e-g).

As an alternative to the genetic approach and one potentially applicable to humans, we have exploited the dependence of senescent cells on specific pro-survival pathways and identified a combination of "senolytics" - dasatinib (D; an FDA-approved tyrosine kinase inhibitor ${ }^{28}$ ) and quercetin (Q; a flavanol present in many fruits and vegetables $\left.{ }^{29}\right)$ - that specifically kill senescent cells without affecting proliferating or quiescent, differentiated cells ${ }^{5,6}$. To test this approach, 20-month-old male C57BL/6 mice were randomized to either vehicle or D+Q treatment once monthly for 4 months (Fig. 2a). D+Q treatment led to significantly lower p16 $6^{\text {Ink4a }}$ mRNA in bone (Fig. 2b) as well as a lower percentage of senescent osteocytes based on the SADS assay (Fig. 2c) in D+Q- versus vehicle-treated mice. The systemic clearance of senescent cells with $\mathrm{D}+\mathrm{Q}$ was verified by demonstrating lower $p 16^{\operatorname{Ink} 4 a} \mathrm{mRNA}$ (Fig. $2 \mathrm{~d}$ ) and SA- $\beta$-Gal positive cells (Fig. $2 \mathrm{e}-\mathrm{g}$ ) in adipose tissue of 24-month-old D+Qtreated mice. As in the INK-ATTAC model, D+Q administration for 4 months resulted in substantially better spine trabecular bone microarchitecture relative to vehicle (Fig. $2 \mathrm{~h}-\mathrm{m}$ ) with similar improvements in trabecular bone at the femur (Supplementary Fig. 10a-f). Similar to the INK-ATTAC model, trabecular bone histomorphometry demonstrated lower osteoclast numbers per bone perimeter in D+Q- versus vehicle-treated mice (Supplementary Fig. 10g) with no differences in osteoblast numbers, mineral apposition rate, or bone formation rate (Supplementary Fig. 10h-j). Also similar to the INK-ATTAC model, D+Q treatment resulted in higher femur cortical thickness and $\mu$ FEA-derived bone strength (Fig. $2 n-p$ ). This was associated with lower osteoclast numbers on the endocortical surface (Fig. 2q) along with higher endocortical osteoblast numbers (Fig. 2r), mineral apposition rate (Fig. 2s), and bone formation rate (Fig. 2t). Thus, both the genetic (INK-ATTAC) and pharmacological (D+Q) approaches for clearing senescent cells led to virtually identical improvements in trabecular and cortical bone microarchitecture with similar underlying cellular mechanisms in trabecular and cortical bone compartments.

Because senescent cell clearance was associated with lower osteoclast numbers and bone resorption in vivo, we next tested the direct effects of senescent cells on osteoclastogenesis. Pre-treatment of whole mouse bone marrow with senescent cell CM resulted in greater osteoclast differentiation potential of non-adherent marrow cells as compared to control CM pre-treatment, as evidenced by the markedly higher number of tartrate-resistant acid phosphatase (TRAP)-positive, multinucleated osteoclasts (Fig. 3a,b). The senescent cell CM-treated osteoclast differentiation cultures also exhibited significantly higher mRNA expression of the osteoclast markers, Ctsk, Oc-stamp, Oscar, Tnfrs11a (Rank), and Acp5 (Trap) (Fig. 3c).

To determine the mechanism for increased osteoclastogenic potential, non-adherent bone marrow cells were assessed following CM pre-treatment. Flow cytometry demonstrated that the percentage of early osteoclast progenitor cells (defined as CD115 [monocyte marker]+/ 
Receptor Activator of NFkB [RANK-]) was higher by over 2-fold in cells exposed to senescent cell versus control CM (Fig. 3d,e). Since RANK expression increases with osteoclast differentiation $^{30}$, these data indicate that senescent cell CM increased the number of early monocyte osteoclast progenitors that had not yet acquired RANK expression. This increase was due to enhanced survival, as apoptosis was significantly lower in whole marrow (Fig. 3f) as well as in monocyte-enriched cultures (Fig. 3g) exposed to senescent cell versus control CM. Senescent cell CM pre-treatment did not alter differentiation of monocyteenriched cultures plated at equal density, indicating no direct effect of senescent cell CM on monocyte differentiation into osteoclasts (Supplementary Fig. 11a).

We have previously shown that the JAKi, ruxolitinib, attenuates the production of multiple SASP components by senescent cells ${ }^{3,7}$, and CM from JAKi-treated senescent cells exhibited a markedly reduced ability to promote osteoclast differentiation as compared to CM from senescent cells (Fig. 3h,i). This effect was due to JAKi effects on senescent cell SASP secretion, since adding the JAKi directly to senescent cell CM did not affect osteoclastogenesis (Supplementary Fig. 11b,c). In our earlier study ${ }^{7}$, we identified IL-6, IL-8 (human homolog to mouse CXCL1), and PAI-1 as the most critical components of the SASP that were markedly downregulated by JAK inhibition; since these cytokines also modulate bone resorption ${ }^{31-33}$, we next used neutralizing antibodies to each of them, which revealed that as compared to an IgG control, neutralization of each of these SASP components attenuated the pro-osteoclastogenic effects of senescent cell CM (Fig. 3j).

In order to test the in vivo effects of SASP inhibition on age-related bone loss, 22-month-old male C57BL/6 mice were treated with the JAKi, ruxolitinib, for two months (Fig. 4a). Relative to vehicle, JAKi-treated mice had better spine trabecular bone microarchitecture (Fig. 4b-g), with similar differences at the femur (Fig. 4h-m). JAKi treatment also resulted in significantly higher bone strength at the femur (Fig. 4n). In contrast, two months treatment of young adult male C57BL/6 mice (7-month-old) with the JAKi did not alter bone parameters (Supplementary Fig. 12), indicating that this strategy is specific for prevention of age-related bone loss. Similar to the trabecular bone histomorphometry findings following the reduction of senescent cells using the INK-ATTAC suicide transgene or following D+Q treatment, trabecular bone histomorphometry in the JAKi-treated old mice revealed significantly lower osteoclast numbers per bone perimeter as compared to vehicletreated mice (Fig. 4o), with no significant differences in osteoblast numbers (Fig. 4p). Ex vivo analysis of bone marrow revealed a significantly lower osteoclast progenitor colony forming units- granulocyte-monocyte (CFU-GM) and -monocytes (CFU-M) (Supplementary Fig. 13a,b). In addition, bone marrow isolated from old JAKi-treated mice had significantly impaired osteoclastogenic potential as compared to vehicle treatment (Supplementary Fig. $13 \mathrm{c}, \mathrm{d})$; consistent with a lack of effect of the JAKi on skeletal parameters in young mice, the osteoclastogenic potential of bone marrow from young vehicle- versus JAKi-treated mice was virtually identical (Supplementary Fig. 13e). Finally, to further validate our in vitro findings, we also measured circulating levels of IL-6, CXCL1 (IL-8), and PAI-1 in the vehicle- versus JAKi-treated mice and as shown in Supplementary Fig. 14a, all three cytokines were lower in the JAKi- versus vehicle-treated mice. By contrast, these cytokines were not lower in plasma from INK-ATTAC (Supplementary Fig. 14b) or D+Q-treated (Supplementary Fig. 14c) mice, suggesting that the JAKi has more systemic effects on the 
SASP whereas changes in SASP factors following the specific elimination of senescent cells may be more localized to their microenvironment.

Age-related cellular senescence has been causally linked to declining cardiovascular and metabolic function ${ }^{3,4}$, as well as frailty ${ }^{7}$. Here, we examine another major aging comorbidity, osteoporosis, and establish the causal role of senescence in age-related bone loss. We use three different approaches to attenuate both the systemic and paracrine detrimental effects of senescent cells in 20- to 22-month-old mice, which have substantial bone loss that mimics osteoporosis in elderly humans. Concomitant with reducing the burden of senescent cells (genetically or pharmacologically) or with inhibiting the production of the SASP by senescent cells (pharmacologically), we demonstrate a significantly better skeletal phenotype relative to vehicle treatment with all three approaches. Using bone histomorphometry, we further demonstrate that targeting senescent cells results in lower bone resorption. Importantly, despite the lower bone resorption, bone formation is either maintained (trabecular bone) or is higher (cortical bone) following reduction of the senescent cell burden. The inhibition of bone resorption without a concomitant reduction, or an actual stimulation, in bone formation resulting from targeting senescent cells is in contrast to all currently available anti-resorptive drugs for osteoporosis (bisphosphonates, denosumab, estrogen, or raloxifene), where a reduction in bone resorption is uniformly associated with a reduction in bone formation ${ }^{34}$. The only other class of drugs to inhibit bone resorption with comparatively lesser effects on bone formation than bisphosphonates or denosumab were the cathepsin $\mathrm{K}$ inhibitors ${ }^{34}$. Unfortunately, development of this pharmacologic class has recently been halted due to an unforeseen increase in stroke risk in a large, phase III odanacatib trial ${ }^{35}$. Importantly, new drugs such as senolytics, which inhibit bone resorption without a concomitant reduction in bone formation, would be expected to be efficacious in treating age-related osteoporosis since the "pure" anti-resorptive bisphosphonate, zoledronic acid, increased bone mineral density and reduced subsequent vertebral and non-vertebral fractures in women and men with a mean age of $\sim 75$ years ${ }^{36}$.

It should be noted that, based on the availability of very old mice from the National Institute on Aging mouse colony, female mice were used in the INK-ATTAC study, whereas male mice were used in the D+Q and JAKi studies. This accounts for the differences in BV/TV in the vehicle-treated mice in the three studies. Since qualitatively similar effects were seen using all three approaches in different sexes, it could be argued that this strengthens our conclusions.

In our previous work ${ }^{16}$, we found that multiple cell types in the bone microenvironment, including hematopoietic cells, had increased $p 16^{I n k 4 a}$ expression with aging. Moreover, not only senescent osteocytes, but also senescent myeloid cells, developed the SASP. Since all three of our approaches were not cell-specific, whether elimination of senescent hematopoietic (e.g., myeloid cells) or senescent mesenchymal cells (e.g., osteocytes) was specifically responsible for the beneficial effects of reducing senescent cell burden or the SASP on skeletal parameters in mice with aging will require further study. In addition, it is possible that the skeletal effects of targeting senescent cells are due to changes occurring outside of bone, through systemic factors such as cytokines, growth factors, or hormones. 
Although clearing senescent cells using a genetic approach is not feasible in humans, the two pharmacological approaches described here are clearly translatable. While JAK inhibition does have effects on multiple tissues ${ }^{7}$ and effects of the JAKi besides SASP inhibition could be contributing to its skeletal effects, suppressing the SASP to enhance bone mass is, indeed, novel and other potentially emerging compounds that have similar actions in bone without deleterious effects in other tissues could be translated to the clinic. Moreover, targeting senescent cells using senolytic drugs is another viable, translatable approach. This is because these drugs would only need to be given intermittently, thereby killing senescent cells without other, off-target effects. Note that in our study, D+Q were administered once monthly, thereby minimizing possible effects related to pathways other than their senolytic effects. In addition, based on the pro-survival pathways previously shown to confer apoptosis resistance to senescent cells ${ }^{5,6}$, additional senolytic agents have been identified, such as the Bcl-2 family inhibitor, navitoclax (ABT263) ${ }^{37}$. Regardless of which specific senolytic compounds are eventually used for osteoporosis and other aging morbidities, our study provides a critical proof-of-concept for the feasibility of this approach for treatment of age-related bone loss.

In conclusion, osteoporosis is an enormous public health problem that will only increase in scope with the aging of the population ${ }^{38}$. In addition to the morbidity associated with fractures, up to $25 \%$ of elderly hip fracture patients die within a year of this devastating event ${ }^{39}$. Thus, there is a critical need to identify novel therapeutic paradigms for age-related bone loss. The approaches identified here for either eliminating senescent cells or suppressing their SASP represent, to our knowledge, the first therapeutic paradigm to treat age-related osteoporosis by targeting not just bone-specific pathways, as with all currently available (or soon to be available) drugs, but rather by targeting a fundamental aging mechanism present in all tissues. Indeed, whereas conventional anti-resorptive therapy predominantly targets osteoclasts to decrease bone resorption with a concomitant decrease in bone formation due to coupling (Fig. 4q), senolytics reduce senescent cells, which in turn suppresses bone resorption with either an increase (cortical bone) or maintenance (trabecular bone) in bone formation (Fig. 4r). Furthermore, eliminating senescent cells or inhibiting the production of their SASP has been shown to improve cardiovascular function ${ }^{4}$, enhance insulin sensitivity ${ }^{3}$, and reduce frailty ${ }^{7}$ in old mice. The present work demonstrates that a similar approach may also be useful to treat osteoporosis, making it fundamentally different from all current therapies for osteoporosis, which only benefit bone. Therefore, therapeutically targeting senescent cells would not only be a novel strategy to treat osteoporosis by simultaneously reducing bone resorption and enhancing bone formation, but also to concurrently prevent multiple aging co-morbidities by targeting a fundamental aging mechanism.

\section{Online Methods}

\section{General experimental approaches}

No samples, mice or data points were excluded from the reported analyses. Animals were randomized to experimental groups as indicated below. Analyses were performed in a 
blinded fashion as noted below. Detailed information on experimental design and reagents is available through the accompanying Life Sciences Reporting Summary.

\section{Mouse strains and drug treatments}

Mice were housed in ventilated cages and maintained within a pathogen-free, accredited facility under a 12-hr light/dark cycle with constant temperature $\left(23^{\circ} \mathrm{C}\right)$ and access to food (standard mouse diet, Lab Diet 5053, St. Louis, MO) and water ad libitum. All animal protocols were approved by the Institutional Animal Care and Use Committee (IACUC), and all experiments were performed in accordance with IACUC guidelines. Both sexes were studied as specified below and in Figure Legends.

The generation and characterization of the INK-ATTAC transgenic mouse line has been described $^{2}$. Based on the original idea and initial experimental strategies devised by J.L.K. and T.T., INK-ATTAC mice were co-developed by the Kirkland and J. van Deursen (Mayo) laboratories, bred onto a C57BL/6 background in the van Deursen laboratory, and then the Kirkland laboratory genotyped them to select INK-ATTAC heterozygotes and aged them to 6-12 (young) or 20 (old) months. All experiments using the INK-ATTAC mice were performed on transgenic line 3 , which contains 13 copies of the transgene inserted into a single locus ${ }^{2}$. Female INK-ATTAC mice from this cohort were randomly assigned to be injected (intraperitoneally [i.p.]) with vehicle or AP20187 (B/B homodimerizer, Clontech; $10 \mathrm{mg}$ of AP20187 per kg body mass [drug/body mass]) twice weekly beginning at 20 months of age for a total of 4 months (all old mice were sacrificed at 24 months of age). This dosing regimen was chosen because it was effective in clearing senescent cells in chronologically aged old $I N K$-ATTAC mice in previous studies ${ }^{3,4}$. Young (12-month) female INK-ATTAC mice were treated using the same dosing regimen for 1.5 months (sacrificed at 13.5 months of age). In addition, from a previous study in the Kirkland laboratory in which no bones were harvested, we obtained plasma samples from young (6-month) female INK$A T T A C$ mice that were treated using the same dosing regimen for 4 months.

C57BL/6 male mice were obtained from the National Institute on Aging (NIA) at 7-, 20- or 22 -months of age. Alternatively, C57BL/6 breeding colonies were maintained to generate animals for in vitro osteoclastogenesis assays. Twenty-month-old male C57BL/6 mice were randomly assigned to once monthly treatments by oral gavage with dasatinib and quercetin $(\mathrm{D}+\mathrm{Q})$ or vehicle for four months ${ }^{5}$. In pilot studies, weekly and bi-weekly D+Q were also evaluated, with similar effects to once monthly dosing. The once monthly dosing regimen was chosen to avoid potential off-target effects and because it was effective in clearing senescent cells in a previous cohort of old mice 4 . D and Q were diluted in 10\% PEG400 and delivered by oral gavage at dosages of $5 \mathrm{mg} / \mathrm{kg}$ and $50 \mathrm{mg} / \mathrm{kg}$, respectively, in $100 \mu \mathrm{L}$.

Seven- (young) or 22-month (old) male C57BL/6 mice were randomly assigned to treatment with either the JAK 1/2 inhibitor, ruxolitinib (JAKi), or vehicle for 2 months (mice sacrificed at 9 months [young] or 24 months [old] of age), as part of a previously published study ${ }^{7}$. JAKi was dissolved in DMSO and mixed with chow. DMSO (vehicle) chow or JAKi chow was administered daily ( $0.5 \mathrm{~g}$ per animal) for two months at a dose of $60 \mathrm{mg} / \mathrm{kg}$ (drug/body mass). This dosing regimen was effective in blocking the SASP in old mice? 
Note that whereas female mice were used in the INK-ATTAC study, based on availability from the National Institute on Aging mouse colony, male mice were used in the senolytic and JAK-inhibitor studies.

\section{Tissue collection}

Mice were sacrificed, body mass was recorded and serum/plasma was collected via cardiac puncture at sacrifice and stored at $-80^{\circ} \mathrm{C}$. The right femur/tibia and lumbar vertebrae were fixed in 10\% neutral buffered formalin and stored in ethanol to be used for micro-computed tomography $(\mu \mathrm{CT})$, histomorphometry, and SADS). The remaining vertebrae were used for osteocyte-enriched cell isolations. The tail was collected at the base and stored in PBS soaked gauze at $-20^{\circ} \mathrm{C}$ for biomechanical compression and biomaterial nanoindentation testing of the caudal vertebrae. For in vitro analyses of osteoclast progenitors or osteoclastogenesis, left femurs/tibiae were isolated and dissected free of soft tissue, epiphyses were removed, and marrow was flushed with PBS. As described ${ }^{7}$, perigonadal adipose tissue was collected and either fixed (for SA- $\beta-$ Gal staining) or snap frozen in liquid nitrogen for RNA and subsequent rt-qPCR analyses.

\section{Isolation of osteocyte-enriched cells}

Detailed methods and validation of our osteocyte-enriched cell isolation protocol are presented elsewhere ${ }^{16,27}$. Briefly, mouse vertebrae were stripped of muscle/connective tissues, minced, and sequentially digested twice for 30-min in collagenase (Liberase; Roche Diagnostics GmbH, Mannheim, Germany). As shown previously ${ }^{16}$, the remaining cell fraction represents a highly enriched population of osteocytes used for rt-qPCR analyses.

\section{SADS analysis of senescent osteocytes}

Senescence-associated distention of satellites (SADS) ${ }^{9}$ were measured in vivo in a blinded fashion as described ${ }^{16}$ in osteocytes located in cortical bone in the long bone diaphyses. Briefly, the decalcified right tibiae of mice were fixed, embedded in methylmethacrylate (MMA), and sectioned, followed by fluorescent in situ hybridization (FISH) ${ }^{16}$. Bone sections were crosslinked with 4\% paraformaldehyde (PFA) for 20 min, washed 3 times in PBS (5 min each), and dehydrated in graded ethanol as follows: 70\%, 90\%, and 100\% (3 min each). Following a brief air drying, sections were denatured for $10 \mathrm{~min}$ at $80^{\circ} \mathrm{C}$ in hybridization buffer: $70 \%$ formamide (Sigma), $25 \mathrm{mM} \mathrm{MgCl}_{2}, 0.1 \mathrm{M}$ Tris (pH 7.2), $5 \%$

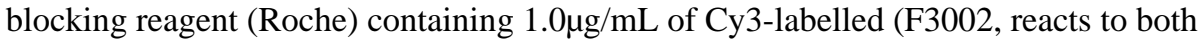
human and mouse), and CENPB-specific (ATTCGTTGGAAACGGGA) peptide nucleic acid (PNA) FISH probe (Panagene Inc, Korea), followed by hybridization for $2 \mathrm{hrs}$ at room temperature in the dark. Slides were washed, stained, and mounted with vectashield DAPIcontaining mounting media (Life Technologies). In addition, we performed the SADS assay on primary murine osteocytes isolated from long-bone diaphyses using the Bonewald Laboratory technique ${ }^{40}$; cells were either untreated or treated with $10 \mathrm{~Gy}$ of cesium irradiation (to induce senescence) and cultured in a humidified incubator (maintained at $37^{\circ} \mathrm{C}$ and $5 \% \mathrm{CO}_{2}$ ) for 20 days. SADS were visualized using confocal microscopy (Mayo Clinic Microscopy and Cell Analysis Core) as described ${ }^{16}$. The number of decondensed/ elongated centromeres per osteocyte were quantified, as described ${ }^{16}$. We previously established a cut-off for defining cell senescence as $\geq 4$ SADS per cell ${ }^{16}$. 


\section{Senescence-associated $\beta$-galactosidase (SA- $\beta$-Gal) assay}

Cellular SA- $\beta-G a l$ activity was measured to detect lysosomes in senescent cells ${ }^{41}$. Detailed methods for the SA- $\beta$-Gal assay have been previously described ${ }^{7}$. Briefly, perigonadal adipose tissue samples or primary murine osteocytes were fixed with $2 \%(\mathrm{vol} / \mathrm{vol})$ formaldehyde (Sigma-Aldrich) and 25\% glutaraldehyde (Sigma-Aldrich) in PBS for 5 min. After washing with PBS 3 times, samples were incubated in SA- $\beta$-Gal solution (pH 6.0) at $37^{\circ} \mathrm{C}$ for $16-18 \mathrm{hrs}$. Ice-cold PBS was then used to stop the enzymatic reaction. In blinded analyses, for each sample, ten images were taken from random fields using Fluorescence Microscopy (Nikon Eclipse Ti). DAPI (Life Technologies) was used to stain nuclei for cell counting.

\section{Skeletal phenotyping}

All imaging was performed in a blinded fashion. Areal bone mineral density (aBMD; $\mathrm{g} / \mathrm{cm}^{2}$ ) of the lumbar spine $\left(\mathrm{L}_{1}-\mathrm{L}_{4}\right)$ was measured by dual-energy X-ray absorptiometry (DXA) using a Lunar PIXImus densitometer (software version 1.44.005; Lunar Corp., Madison, $\mathrm{WI})^{42}$. Measures of total (metaphysis), trabecular (metaphysis), and cortical (midshaft diaphysis) volumetric BMD (vBMD; $\mathrm{mg} / \mathrm{cm}^{3}$ ) at the tibia were obtained using peripheral quantitative computed tomography (pQCT; Stratec XCT Research SA Plus, software v5.40, Nordland Medical Systems, Fort Atkinson, WI) ${ }^{42}$. Quantitative analysis of the lumbar spine (L4-L6) and distal femoral metaphysis were performed using the Viva Scan $40 \mu \mathrm{CT}$ scanner (Scanco Medical AG, Basserdorf, Switzerland) with the following parameters: $55 \mathrm{kVp}$, $145 \mathrm{~mA}$, high resolution, 21.5 diameter, $10.5 \mu \mathrm{m}$ voxel size, $300 \mathrm{~ms}$ integration time. Using two-dimensional (2D) data from scanned slices, 3D analysis was used to calculate morphometric parameters at both the lumbar spine (200 slices) and distal femoral metaphysis (100 slices) defining trabecular bone mass and microarchitecture, including trabecular bone volume fraction (BV/TV; \%), trabecular number (Tb.N; 1/mm), trabecular thickness (Tb.Th; $\mathrm{mm}$ ), trabecular separation (Tb.Sp; $\mathrm{mm}$ [higher values are associated with weaker bone]), and the structure model index (SMI), which indicates whether trabeculae are stronger, plate-like (lower values) or weaker, rod-like (higher values). Cortical thickness (Ct.Th; $\mathrm{mm}$ ) was assessed at the distal femoral metaphysis (50 slices). Micro-finite element analysis $(\mu \mathrm{FEA})$ was performed at the femoral metaphysis to assess failure load (N; i.e., bone strength) using the manufacture's software (Scanco Medical AG, Basserdorf, Switzerland; Finite Element-Software Version 1.13). All $\mu$ CT parameters were derived using the manufacturer's protocols.

\section{Compression loading}

Loading tests were performed in a blinded fashion. The sixth caudal vertebrae $(\mathrm{Ca} 6)$ was removed from the tail, stripped of soft tissue, and measured end-to-end with a digital caliper (ABSOLUTE, Mitutoyo, Aurora, IL). Vertebrae were soaked in PBS for 15 to 30 minutes before testing to ensure hydration. Cyanoacrylate adhesive was applied to each end of the bones to mount them between two \#10-32 stainless steel nuts filled with PMMA resin. The assembly was loaded into an alignment guide to impart parallel loading surfaces at each end of the bone while the adhesive cured for five minutes. Compression testing was conducted with a servohydraulic test system instrumented with a 450-Newton (N) capacity load cell 
(Mini Bionix II, MTS Systems, Eden Prairie, MN). Bones were pre-loaded to $1 \mathrm{~N}$ and then loaded until failure under displacement control at a rate of $0.02 \mathrm{~mm} / \mathrm{s}$, as previously described ${ }^{24}$. Force and displacement data were collected at a sample rate of $100 \mathrm{~Hz}$. The yield load $(\mathrm{N})$, ultimate load $(\mathrm{N})$, ultimate displacement $(\mathrm{mm})$, and energy to ultimate failure $(\mathrm{mJ})$ were evaluated.

\section{Nanoindentation}

Nanoindentation was performed in a blinded fashion. The seventh caudal vertebra (Ca7) was removed from the tail and stripped of soft tissue. Specimens were embedded in PMMA resin (Lecoset 100, Leco, St. Joseph, MO) and then sectioned transversely within the body of the vertebrae with a low-speed diamond saw (Isomet, Buehler, Lake Bluff, IL). Sections were manually polished with a polishing/griding system (Ecomet 250, Buehler) using successively finer abrasive cloths (400, 600, 800, and 1200 grit) with a final polish using a microcloth and slurry of $0.05 \mu \mathrm{m}$ aluminum abrasive (Union Carbide, Houston, TX). Indentation testing was conducted on cortical bone with a nanoindentation system (TI 950, Hysitron, Minneapolis, MN) equipped with a diamond Berkovitch pyramidal tip. Four sites, widely distributed around cross-section, were tested on each bone. At each site, a $2 \times 2$ array was indented with $15 \mu \mathrm{m}$ spacing between indents. Indentation was conducted under load control at a rate of $500 \mu \mathrm{N} / \mathrm{s}$ to a peak load of $2000 \mu \mathrm{N}$ with a $60 \mathrm{~s}$ hold before unloading to reduce viscoelastic effects, as previously described ${ }^{26}$. The reduced modulus $\left(\mathrm{E}_{\mathrm{r}} ; \mathrm{GPa}\right)$, and hardness (H; GPa), were calculated using the Oliver-Pharr model, as previously described ${ }^{25}$. Measures were averaged over the four indents of the array to generate a value for the site. Values obtained at the four sites were averaged to generate an aggregate value for each specimen.

\section{Histomorphometric analyses}

All histomorphometric analyses were performed in a blinded fashion. For dynamic histomorphometric analyses, mice were injected subcutaneously with Alizarin Red $(0.1 \mathrm{~mL} /$ animal, $7.5 \mathrm{mg} / \mathrm{mL})$ and calcein $(0.1 \mathrm{~mL} /$ animal, $2.5 \mathrm{mg} / \mathrm{mL})$ on days 9 and 2, respectively, prior to euthanasia. The lumbar vertebrae and right femur were isolated from female $I N K$ ATTAC mice treated with vehicle or AP20187 or male C57BL/6 mice treated with vehicle or $\mathrm{D}+\mathrm{Q}$ and were embedded in MMA, sectioned, and stained with Masson Trichrome to assess osteoblast numbers per bone perimeter (N.Ob/B.Pm, $/ \mathrm{mm}$ ), or stained for tartrate-resistant acid phosphatase (TRAP) activity to assess osteoclast numbers per bone perimeter, N.Oc/ B.Pm, $/ \mathrm{mm})$. Alternatively, sections were left unstained to quantify mineralizing surfaces (mineral apposition rate, MAR, $\mathrm{mcm} / \mathrm{d}$; bone formation rate per bone surface, BFR/BS, $\mathrm{mcm}^{3} / \mathrm{mcm}^{2} / \mathrm{d}$ ). Femurs obtained from vehicle or JAKi-treated C57BL/6 male mice were decalcified for two weeks in $12.5 \%$ EDTA, followed by paraffin embedding, sectioning, and staining with Goldner's Trichrome stain. Osteoblast (N.Ob/B.Pm) and osteoclast (N.Oc/ B.Pm) numbers were assessed. The determination of bone marrow adipocyte parameters has been described by our group previously ${ }^{42}$. Briefly, adipocyte number, adipocyte perimeter $(\mathrm{mm})$, and adipocyte volume per tissue volume were measured by tracing out individual adipocytes in all the fields analyzed. All histomorphometric measurements and calculations were performed with the Osteomeasure Analysis system (Osteometrics, Atlanta, Georgia). 


\section{Real-time quantitative polymerase chain reaction (rt-qPCR) analyses}

Targeted gene expression analyses were performed by rt-qPCR as described ${ }^{16}$. Briefly, total RNA was isolated using QIAzol Lysis Reagent and RNeasy Mini Columns (QIAGEN, Valencia, CA). DNase treatment was applied to degrade contaminating genomic DNA using an on-column RNase-free DNase solution (QIAGEN, Valencia, CA). RNA quantity and purity were confirmed with a Nanodrop spectrophotometer (Thermo Scientific, Wilmington, DE). Reverse transcriptase was performed using the High-Capacity cDNA Reverse Transcription Kit (Applied Biosystems by Life Technologies, Foster City, CA). PCR reactions were run using the ABI Prism 7900HT Real Time System (Applied Biosystems, Carlsbad, CA) with either SYBR green (QIAGEN, Valencia, CA) or TaqMan (Applied Biosystems, Carlsbad, CA). Note that TaqMan assays (purchased from Thermo Fisher Scientific) were used to measure $p 16^{\operatorname{Ink} 4 a}$ [Cdkn2a], EGFP, Sost, Opg [Tnfrsf11b], and Rankl[Tnfsf11] (normalized to Tbp or Actb), whereas all other genes (normalized to TubaI) were measured using SYBR green. Murine primers for Trap (Acp5), Cathepskin K(Ctsk), Oscar, Oc-stamp, and Tnfrsf11a (Rank) were designed using Primer Express ${ }^{\circledR}$ Software Version 3.0 (Applied Biosystems, Foster City, CA) according to the following forward and reverse pair sequences: Acp5: forward 5'-CGCTTCAAAATTCCACGTACAA-3' \& reverse $5^{\prime}$-CAGCATCACTGTGTCCAGCAT-3'; Ctsk: forward 5' GGATGAAATCTCTCGGCGTTT-3' \& reverse 5'-GGTTATGGGCAGAGATTTGCTT-3'; Oscar: forward $5^{\prime}$-CTCTTCAAAAGTGGCCTTGTCA-3' \& reverse $5^{\prime}$ -

GGAAGAACTCAGCCAGCTCAA-3'; Oc-stamp: forward 5' TGTAGCCTGGGCTCAGAAGT- $3^{\prime}$ \& reverse $5^{\prime}$-GTTGGTTGAGGACGAAGAGG-3'; Tnfrsf 11a: forward 5'-ACTGAGGAGACCACCCAAGGA-3' \& reverse $5^{\prime}$ TGAAGAGGAGCAGAACGATGAG-3'.

\section{Conditioned medium generation}

Subcutaneous adipose tissue samples were collected from healthy, lean (BMI 26.6 \pm 0.9 $\mathrm{kg} / \mathrm{m}^{2}$ ) kidney donors aged $39 \pm 3.3$ years for isolation of primary human adipose-derived mesenchymal stem cells (AdMSCs) as described ${ }^{43}$. The protocol was approved by the Mayo Foundation Institutional Review Board informed consent was obtained from all human subjects. Cellular senescence was induced in AdMSCs by 10 Gy of cesium irradiation as described $^{7}$. JAK inhibitor 1 (CAS 457081-03-7) was purchased from EMD Millipore (Billerica, MA). Ruxolitinib (INCB18424, CAS 941678-49-5) was purchased from ChemieTek (Indianapolis, IN). Conditioned medium (CM) was made of RMPI 1640 containing $1 \mathrm{mM}$ sodium pyruvate, $2 \mathrm{mM}$ glutamine, MEM vitamins, MEM non-essential amino acids, and antibiotic (Thermo Fisher Scientific). For collection, human nonproliferating control (CON) or senescent (SEN) AdMSCs cells were washed 3 times with PBS and cultured in CM for 24 hrs. For JAK inhibitor treatment, cells were treated with $0.6 \mu \mathrm{M}$ JAK inhibitor 1 or DMSO in aMEM containing 10\% FBS and antibiotic for 48 hrs. Cells were then washed 3 times with PBS and exposed to media containing JAK inhibitor or DMSO for another $24 \mathrm{hrs}$. 


\section{In vitro osteoblast differentiation}

MC3T3-E1 (subclone 4) cells were plated at a density of $1 \times 10^{4}$ cells per $\mathrm{cm}^{2}$ in aMEM without ascorbic acid. After reaching confluency ( 48 hours), media was replaced and cells were cultured for an additional 72 hours. Differentiation media consisted of aMEM containing ascorbic acid with the addition of $50 \mu \mathrm{g} / \mathrm{mL}$ ascorbic acid and $10 \mathrm{mM}$ betaglycerophosphate. Cells were cultured in the presence of $20 \%$ control or senescent CM. Differentiation media and CM treatments were replaced every 3-4 days until day 21. Cells were fixed with $1 \%$ PFA, and stained with Alizarin Red S (Sigma) to assess osteoblast mineralization. Cultures were stained for 15 minutes, washed with $\mathrm{H}_{2} \mathrm{O}$, and dried before obtaining images. Alizarin Red stain was eluted with $10 \%$ cetylpyridinium chloride in $\mathrm{NaP}$ buffer and absorbance was assessed at $540 \mathrm{~nm}$.

\section{Osteoclast differentiation and quantification}

Following euthanasia, long bones were isolated from C57BL/6 mice, 5-8 weeks of age. As described above, bone marrow was flushed with sterile PBS. Red blood cells (RBCs) were lysed using RBC lysis buffer (1 $\times$ ) (eBioscience, San Diego, CA), and marrow counts were obtained. Equal cell numbers were cultured overnight in vehicle (negative control), $25 \mathrm{ng} / \mathrm{mL}$ rmM-CSF (positive control) (R\&D Systems, Minneapolis, MN), control CM, senescent CM, or CM from senescent cells treated with the JAK inhibitor 1 (SEN/JAKi). CM treatments were 1:1 with a-Minimal Essential Medium (MEM, Invitrogen, Carlsbad, CA) with 10\% fetal bovine serum (FBS) and $1 \times$ antibiotic/antimycotic. Negative and positive controls were 1:1 with control RPMI 1640 used for AdMSC culture. As a control for the presence of the JAK inhibitor 1, cells were treated with control $\mathrm{CM}$ or senescent $\mathrm{CM}$ with the addition of freshly added vehicle (DMSO) or JAK inhibitor $1(0.3 \mu \mathrm{M}$ final concentration). The nonadherent populations were collected $24 \mathrm{hrs}$ after plating. Cell counts were obtained, and viability was assessed with Trypan Blue. Equal cell numbers were then plated in osteoclast differentiation medium [aMEM with 10\% FBS, 1× antibiotic/antimycotic, 1:50 CMB 14-12

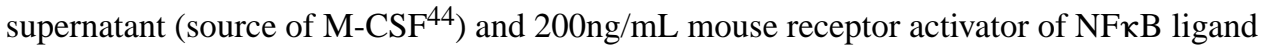
(RANKL)-GST (generated using a recombinant RANKL expression construct provided by Dr. Beth Lee, Ohio State University)]. Osteoclast differentiation was carried out for two to four days, with differentiation media replaced on day three. Mature osteoclasts formed on days three and four.

Alternatively, mouse monocytes were isolated from mouse bone marrow by negative selection using the Mouse Monocyte Isolation Kit (Miltenyi Biotec, Inc., San Diego, CA) and magnetic activated cell sorting (MACS, autoMACS-Pro magnetic cell sorter, Miltenyi Biotec, Inc.). Monocytes were cultured with CON or SEN CM overnight, as described above. Non-adherent populations were collected, counted, and plated at equal cell densities in osteoclast differentiation medium.

To assess osteoclastogenesis in vehicle or ruxolitinib (JAKi) treated mice, RBC-lysed marrow was plated directly in osteoclast differentiation medium. Osteoclast differentiation medium was replaced on day three and osteoclast cultures were fixed on day four. 
Mature osteoclast cultures were fixed in 1\% PFA and osteoclast differentiation was assessed by TRAP activity (Acid Phosphatase, Leukocyte Kit, Sigma, St. Louis, MO) ${ }^{45}$. Osteoclasts were defined as TRAP positive cells with greater than three nuclei.

\section{Flow cytometry}

Whole bone marrow was cultured overnight with CON or SEN CM as described above. The non-adherent cells were collected and counted. For each sample, $5 \mu \mathrm{L} \mathrm{Fc} \mathrm{Blocking} \mathrm{Reagent}$ was added to $1 \times 10^{6}$ cells in FACS buffer, and cells were stained with APC-conjugated antiCd115 (eBioscience) and PE-conjugated anti-RANK (ThermoFisher) in a total volume of $100 \mu \mathrm{L}$. Cells were washed and fixed with $1 \%$ PFA. Cells were sorted using FACSCalibur (BD Biosciences) and data were analyzed by FlowJo (FlowJo, LLC); gates were set according to unstained and single label controls for APC and PE, respectively.

\section{Apoptosis}

Apoptosis was assessed using ApoTox-Glo ${ }^{\mathrm{TM}}$ Triplex Assay (Promega, Madison, WI). Bone marrow or monocyte-enriched cultures (Mouse Mouse Isolation Kit, Miltenyi Biotec) were treated for $24 \mathrm{hrs}$ with CON or SEN CM, and apoptosis was quantified. Alternatively, bone marrow was differentiated for four days into mature osteoclasts as described above. On day four, mature osteoclast cultures were treated with vehicle or 10nM AP20187 (the same in vitro dose used previously ${ }^{2}$ ) in fresh osteoclast differentiation media. Cells were incubated for $18 \mathrm{hrs}$ followed by apoptosis quantification. Briefly, a luminogenic caspase-3/7 substrate was added to cells. Cultures were incubated for $30 \mathrm{~min}$ at RT, and luminescence was measured using the GloMax-Multi Detection System (Promega).

\section{MethoCult assay}

Bone marrow was plated in MethoCult ${ }^{\mathrm{TM}}$ GF 3434 Media (StemCell Technologies, Vancouver, BC, Canada). Each mouse was tested in duplicate. Cultures were incubated for 10 days and colonies were identified as monocyte/macrophage (M) or granulocytemonocyte/macrophage (GM). Colony-forming units (CFU)-M and CFU-GM per plate were counted and averaged per mouse. Analyses were done in a blinded fashion.

\section{Serum/plasma assays}

Peripheral blood for serum/plasma measurements was collected from overnight fasted mice by cardiac puncture; samples were stored at $-80^{\circ} \mathrm{C}$ in aliquots. Circulating plasma $\mathrm{C}$ terminal telopeptide of type I collagen (CTx) levels in young and old INK-ATTAC mice were measured by enzyme immunoassay (EIA) (Immunodiagnostic Systems). Serum IL-6 and CXCL1 (IL-8) levels in JAKi-treated mice were previously measured ${ }^{7}$ using Luminex xMAP technology. Mouse ELISA kits (R\&D Systems) were used to measure circulating plasma levels of IL-6, CXCL1 (mouse homolog to IL-8), and PAI-1 in all the other mice in Supplementary Fig. 14. All assays were performed in a blinded fashion.

\section{Cell line identity}

MC3T3-E1 (subclone 4) cells were obtained from ATCC (ATCC CRL-2593) to assess the effects of senescent $\mathrm{CM}$ on osteoblast differentiation. This cell line is not listed in the 
database of commonly misidentified cell lines maintained by ICLAC. As these cells were freshly obtained from ATCC, we did not perform testing for mycoplasma contamination. All other experiments were performed with primary cells.

\section{Reagents}

Antibodies and other reagents were purchased from commercial sources; details are available from vendors as indicated throughout the Methods.

\section{Statistical analyses}

Sample sizes were based on pilot or previously conducted and published experiments (e.g., Syed et al. ${ }^{42}$ ) in which statistically significant differences were observed on bone with various interventions in our laboratory. For each experiment, replicates are noted in the Figure Legends. All samples presented represent biological replicates. No samples were excluded from analyses. Investigators were blinded to allocation during experiments and outcome assessments, as noted specifically above. Data were checked for normality using histograms and all variables were tested for skewness and kurtosis. Analyses of differences between groups were performed by independent samples $t$-test, Wilcoxon rank-sum tests, one-way ANOVA, or repeated-measures ANOVA where justified as appropriate (see Figure Legends). Following ANOVA, the Bonferroni post-hoc test was used to adjust for multiple comparisons. Data are presented as Means \pm SEM (unless otherwise specified) with $P<0.05$ (two-tailed) considered statistically significant. Statistical analyses were performed using the Statistical Package for the Social Sciences for Windows, Version 22.0 (SPSS, Chicago, IL).

\section{Data availability}

Data presented in this manuscript are available from the corresponding authors upon reasonable request.

\section{Supplementary Material}

Refer to Web version on PubMed Central for supplementary material.

\section{Acknowledgments}

This work was supported by NIH Grants P01 AG004875 (S.K.), R01 AG048792 (S.K.), K01 AR070241 (J.N.F.), K01 AR070281 (M.M.W.), R01 AR068275 (D.G.M.), R37 AG013925 (J.L.K.), AG R21 049182 (J.L.K.), the Connor Group, the Noaber, and the Ted Nash Foundations (J.L.K.), the Glenn Foundation (J.L.K., N.K.L.), and both a High-Risk Pilot Award (J.N.F., S.K.) and Career Development Awards (J.N.F., M.M.W.) from the Mayo Clinic Robert and Arlene Kogod Center on Aging, as well as the Richard F. Emslander Career Development Award in Endocrinology (J.N.F.), the James A. Ruppe Career Development Award in Endocrinology (M.M.W.), and the Glenn/American Federation for Aging Research Postdoctoral Fellowship for Translational Research on Aging (M.X.). We thank Ming Ruan, Glenda L. Evans, Brianne S. Thicke, and James M. Peterson for their technical assistance. We also thank Andrew R. Thoreson, Alexander W. Hooke, and the Mayo Clinic Materials and Structural Testing Resource Laboratory for performing the bone biomechanical compression and nanoindentation testing.

\section{References}

1. Tchkonia T, Zhu Y, van Deursen J, Campisi J, Kirkland JL. Cellular senescence and the senescent secretory phenotype: therapeutic opportunities. J Clin Invest. 2013; 123:966-972. [PubMed: 23454759] 
2. Baker DJ, et al. Clearance of p16Ink4a-positive senescent cells delay aging-associated disorders. Nature. 2011; 479:232-236. [PubMed: 22048312]

3. Xu M, et al. Targeting senescent cells enhances adipogenesis and metabolic function in old age. Elife. 2015; 4:e12997. [PubMed: 26687007]

4. Roos CM, et al. Chronic senolytic treatment alleviates established vasomotor dysfunction in aged or atherosclerotic mice. Aging Cell. 2016; 15:973-977. [PubMed: 26864908]

5. Zhu Y, et al. The Achilles' heel of senescent cells: from transcriptome to senolytic drugs. Aging Cell. 2015; 14:644-658. [PubMed: 25754370]

6. Kirkland JL, Tchkonia T. Clinical strategies and animal models for developing senolytic agents. Exp Gerontol. 2015; 68:19-25. [PubMed: 25446976]

7. Xu M, et al. JAK inhibition alleviates the cellular senescence-associated secretory phenotype and frailty in old age. Proc Natl Acad Sci USA. 2015; 112:301-310.

8. LeBrasseur NK, Tchkonia T, Kirkland JL. Cellular senescence and the biology of aging, disease, and frailty. Nestle Nutr Inst Workshop Ser. 2015; 83:11-18. [PubMed: 26485647]

9. Swanson EC, Manning B, Zhang H, Lawrence JB. Higher-order unfolding of satellite heterochromatin is a consistent and early event in cell senescence. J Cell Biol. 2013; 203:929-942. [PubMed: 24344186]

10. Zhu Y, Armstrong JL, Tchkonia T, Kirkland JL. Cellular senescence and the senescent secretory phenotype in age-related chronic diseases. Curr Opin Clin Nutr Metab Care. 2014; 17:324-328. [PubMed: 24848532]

11. Campisi J, d'Adda di Fagagna F. Cellular senescence: when bad things happen to good cells. Nat Rev Mol Cell Biol. 2007; 8:729-740. [PubMed: 17667954]

12. Campisi J. Senescent cells, tumor suppression, and organismal aging: good citizens, bad neighbors. Cell. 2005; 120:513-522. [PubMed: 15734683]

13. Jurk D, et al. Postmitotic neurons develop a p21-dependent senescence-like phenotype driven by a DNA damage response. Aging Cell. 2012; 11:996-1004. [PubMed: 22882466]

14. Jurk D, et al. Chronic inflammation induces telomere dysfunction and accelerates ageing in mice. Nat Commun. 2014; 2:4172. doi:4110.1038/ncomms5172. [PubMed: 24960204]

15. Minamino T, et al. A crucial role for adipose tissue p53 in the regulation of insulin resistance. Nat Med. 2009; 15:1082-1087. [PubMed: 19718037]

16. Farr JN, et al. Identification of senescent cells in the bone microenvironment. J Bone Miner Res. 2016; 31:1920-1929. [PubMed: 27341653]

17. Wang E. Senescent human fibroblasts resist programmed cell death, and failure to suppress bcl 2 is involved. Cancer Res. 1995; 55:2284-2292. [PubMed: 7757977]

18. Nelson G, et al. A senescent cell bystander effect: senescence-induced senescence. Aging Cell. 2012; 11:345-349. [PubMed: 22321662]

19. Coppe JP, Desprez PY, Krtolica A, Campisi J. The senescence-associated secretory phenotype: the dark side of tumor suppression. Annu Rev Pathol. 2010; 5:99-118. [PubMed: 20078217]

20. Acosta JC, et al. A complex secretory program orchestrated by the inflammasome controls paracrine senescence. Nat Cell Biol. 2013; 15:978-990. [PubMed: 23770676]

21. Herbig U, Ferreira M, Condel L, Carey D, Sedivy JM. Cellular senescence in aging primates. Science. 2006; 311:1257. [PubMed: 16456035]

22. Hamrick MW, et al. Age-related loss of muscle mass and bone strength in mice is associated with a decline in physical activity and serum leptin. Bone. 2006; 39:845-853. [PubMed: 16750436]

23. Glatt V, Canalis E, Stadmeyer L, Bouxsein ML. Age-related changes in trabecular architecture differ in female and male C57BL/6J mice. J Bone Miner Res. 2007; 22:1197-1207. [PubMed: 17488199]

24. Silva MJ, Brodt MD, Uthgenannt BA. Morphological and mechnical properties of caudal vertebrae in the SAMP6 mouse model of senile osteoporosis. Bone. 2004; 35:425-431. [PubMed: 15268893]

25. Oliver WC, Pharr GM. An improved technique for determining the hardness and elastic modulus using load and displacement sensing indentation experiments. J Materials Res. 1992; 7:15641583. 
26. McGee-Lawrence ME, et al. Histone deacetylase 3 is required for maintenance of bone mass during aging. Bone. 2013; 52:296-307. [PubMed: 23085085]

27. Qing H, et al. Demonstration of osteocytic perilacunar/canalicular remodeling in mice during lactation. J Bone Miner Res. 2012; 27:1018-1029. [PubMed: 22308018]

28. Yi JS, et al. Low-dose dasatinib rescues cardiac function in Noonan syndrome. JCI Insight. 2016; 1(20):e90220. [PubMed: 27942593]

29. D'Andrea G. Quercetin: A flavonol with multifaceted therapeutic applications? Fitoterapia. 2015; 106:256-271. [PubMed: 26393898]

30. Arai F, et al. Commitment and differentiation of osteoclast precursor cells by the sequential expression of c-Fms and receptor activator of nuclear factor kappaB (RANK) receptors. J Exp Med. 1999; 190:1741-1754. [PubMed: 10601350]

31. Bellido T, et al. Regulation of interleukin-6, osteoclastogenesis, and bone mass by androgens. $\mathrm{J}$ Clin Invest. 1995; 95:2886-2895. [PubMed: 7769130]

32. Bendre MS, et al. Interleukin- 8 stimulation of osteoclastogenesis and bone resorption is a mechanism for the increased osteolysis of metastatic bone disease. Bone. 2003; 33:28-37. [PubMed: 12919697]

33. Daci E, Verstuyf A, Moermans K, Bouillon R, Carmeliet G. Mice lacking the plasminogen activator inhibitor 1 are protected from trabecular bone loss induced by estrogen deficiency. $\mathrm{J}$ Bone Miner Res. 2000; 15:1510-1516. [PubMed: 10934649]

34. Khosla S. Odanacatib: location and timing are everying. J Bone Miner Res. 2012; 27:506-508. [PubMed: 22354850]

35. Mullard A. Merck \& Co. drops osteoporosis drug odanacatib. Nat Rev Drug Discov. 2016; 15:669.

36. Lyles KW, et al. Zoledronic acid and clinical fractures and mortality after hip fracture. N Engl J Med. 2007; 359:1799-1809.

37. Zhu Y, et al. Identification of a novel senolytic agent, navitoclax, targeting the Bcl-2 family of antiapoptotic factors. Aging Cell. 2016; 15:428-435. [PubMed: 26711051]

38. Wright NC, et al. The recent prevalence of osteoporosis and low bone mass in the United States based on bone mineral density at the femoral neck or lumbar spine. J Bone Miner Res. 2014; 29:2520-2526. [PubMed: 24771492]

39. Burge R, et al. Incidence and economic burden of osteoporosis-related fractures in the United States, 2005-2025. J Bone Miner Res. 2007; 22:465-475. [PubMed: 17144789]

40. Stern AR, et al. Isolation and culture of primary osteocytes from the long bones of skeletally mature and aged mice. Biotechniques. 2012; 52:361-373. [PubMed: 22668415]

41. Lee BY, et al. Senescence-associated beta-galactosidase is lysosomal beta-galactosidase. Aging Cell. 2006; 5:187-195. [PubMed: 16626397]

42. Syed FA, et al. Skeletal effects of estrogen are mediated by opposing actions of classical and nonclassical estrogen receptor pathways. J Bone Miner Res. 2005; 20:1992-2001. [PubMed: 16234973]

43. Tchkonia T, et al. Abundance of two human preadipocyte subtypes with distinct capacities for replication, adipogenesis, and apoptosis varies among fat depots. Am J Physiol Endocrinol Metab. 2005; 288:E267-277. [PubMed: 15383371]

44. Takeshita S, Kaji K, Kudo A. Identification and characterization of the new osteoclast progenitor with macrophage phenotypes being able to differentiate into mature osteoclasts. J Bone Miner Res. 2000; 15:1477-1488. [PubMed: 10934646]

45. Gingery A, Bradley E, Shaw AC, Oursler MJ. Phosphatidylinositol 3-kinase coordinately activates the MEK/ERK and AKT/NFkappaB pathways to maintain osteoclast survival. J Cell Biochem. 2003; 89:165-179. [PubMed: 12682917] 


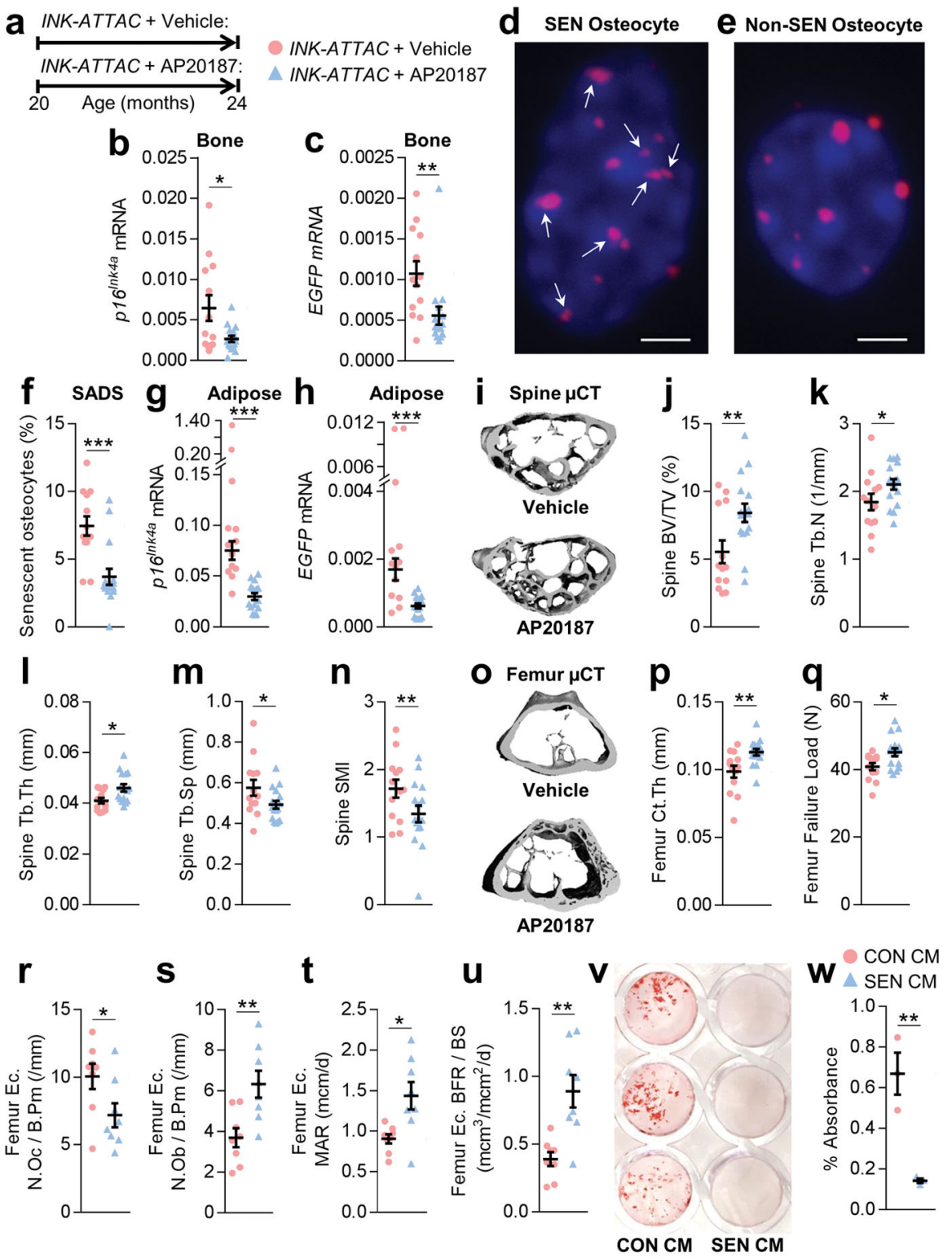

Fig. 1.

Clearance of $p 16^{\text {Ink4at }}$ senescent cells prevents age-related bone loss. (a) Experimental design for testing the effect of senescent cell clearance using a transgenic approach on agerelated bone loss: 20-month-old female INK-ATTAC mice were randomized to either vehicle ( $n=13)$ or AP20187 ( $n=16)$ treatments (intraperitoneally [i.p] twice weekly) for 4 months. rt-qPCR analysis of (b) $p 16^{I n k 4 a}$ and (c) EGFP (encoded by the INK-ATTAC transgene) mRNA expression levels in osteocyte-enriched cells derived from bones of the mice. Representative images ( $n>30$ images per animal, 13 vehicle- and 16 AP20187treated) of $(\mathbf{d})$ a senescent $(\mathrm{SEN})$ osteocyte (magnification $\times 100)$ versus $(\mathbf{e})$ a non-senescent (non-SEN) osteocyte (magnification $\times 100$ ) according to the senescence-associated distension of satellites (SADS, see arrows [in $\mathbf{d}$ ]) assay in cortical bone diaphysis (scale bars, $2 \mu \mathrm{m}$ ). (f) Quantification of the percentage of senescent osteocytes in mice treated with 
either vehicle or AP20187 according to the SADS assay. rt-qPCR analysis of (g) p16 $6^{\text {Ink4a }}$ and (h) EGFPmRNA expression levels in perigonadal adipose tissue. (i) Representative micro-computed tomography $(\mu \mathrm{CT})$ images ( $n=13$ vehicle- and 16 AP20187-treated mice) of bone microarchitecture at the lumbar spine of vehicle- versus AP20187-treated mice. Quantification of $\mu \mathrm{CT}$-derived (j) bone volume fraction (BV/TV; \%), (k) trabecular number (Tb.N; 1/mm), (l) trabecular thickness (Tb.Th; mm), (m) trabecular separation (Tb.Sp; mm), and (n) structure model index (SMI, a measure of plate/rod morphology, with lower numbers being better) at the lumbar spine. (o) Representative $\mu \mathrm{CT}$ images ( $n=13$ vehicle- and 16 AP20187-treated mice) of bone microarchitecture at the femur. Quantification of $\mu \mathrm{CT}$ derived (p) cortical thickness (Ct.Th, $\mathrm{mm})$ and (q) micro-finite-element analysis $(\mu \mathrm{FEA})$ derived failure load (N, Newton [i.e., a measure of bone strength]). Histomorphometric quantification at the femoral endocortical surface of (r) osteoclast numbers per bone perimeter (N.Oc/B.Pm;/mm), (s) osteoblast numbers per bone perimeter (N.Ob/B.Pm;/mm), (t) endocortical mineral apposition rate (MAR; $\mathrm{mcm} / \mathrm{d})$, and (u) endocortical bone formation rate per bone surface (BFR/BS; $\left.\mathrm{mcm}^{3} / \mathrm{mcm}^{2} / \mathrm{d}\right)(n=8 /$ group). (v) Mineralization of osteoblastic MC3T3 cells exposed to control (CON) or senescent (SEN) conditioned medium (CM) ( $n=3$ /group), with quantification of eluted alizarin red dye in (w). Data represent Mean \pm SEM (error bars). $* P<0.05 ; * * P<0.01 ; * * * P<0.001$ (independent samples $t$-test or Wilcoxon rank-sum test, as appropriate). 
a

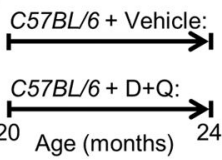

C57BL $6+$ Vehicle $\triangle \mathrm{C} 57 \mathrm{BL} / 6+\mathrm{D}+\mathrm{Q}$
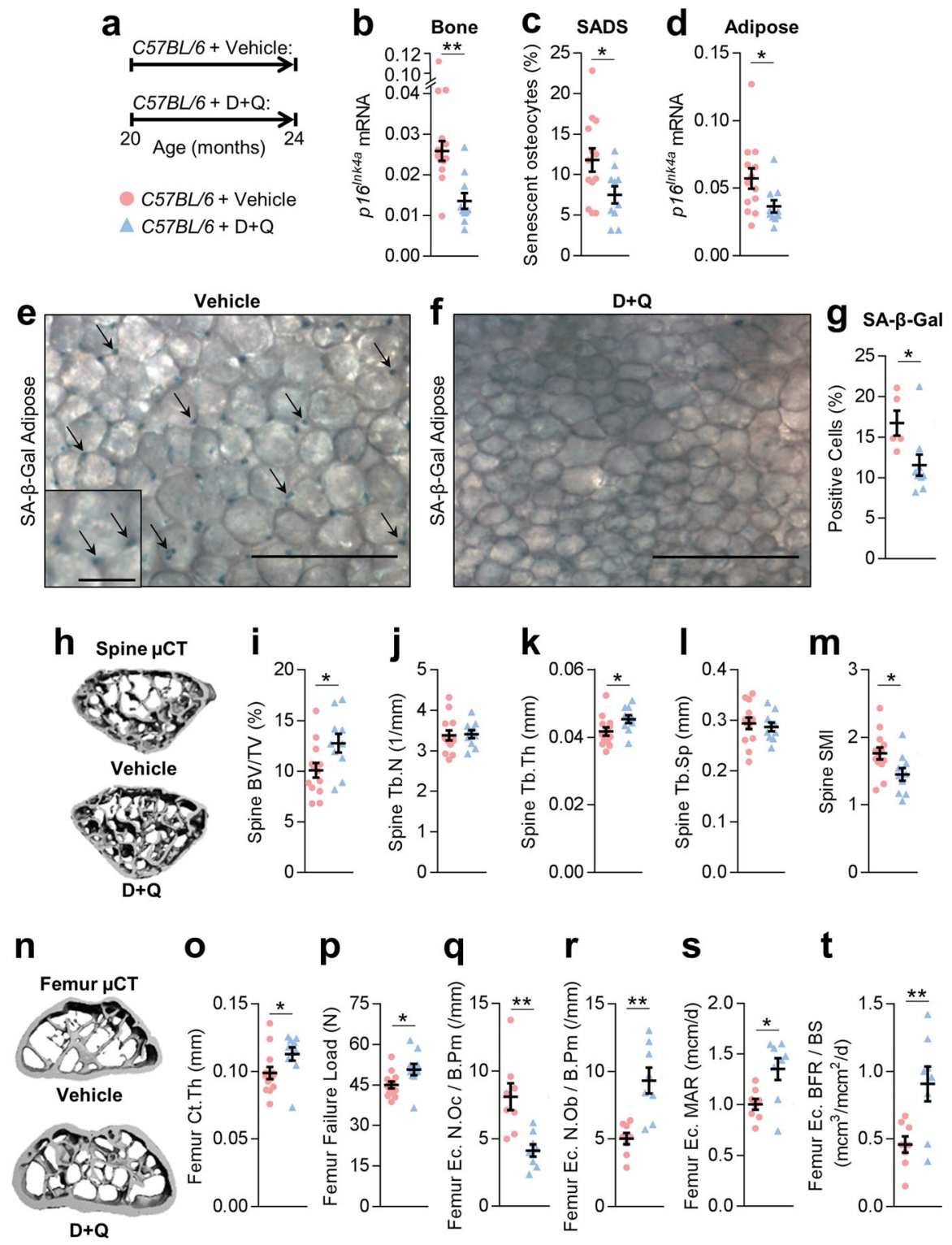

S

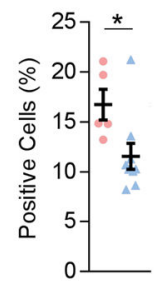

Fig. 2.

Senescent cell clearance by treatment with senolytics (dasatinib + quercetin [D+Q]) prevents age-related bone loss. (a) Experimental design for testing the effect of senescent cell clearance via periodic treatment with $\mathrm{D}+\mathrm{Q}$ on age-related bone loss: 20-month-old male C57BL/6 mice were randomized to either vehicle $(n=13)$ or $\mathrm{D}+\mathrm{Q}(n=10)$ treatments (once monthly by oral gavage) for 4 months. (b) rt-qPCR analysis of $p 16^{\text {Ink } 4 a}$ mRNA expression levels in osteocyte-enriched cells derived from bones, and (c) quantification of the percentage of senescent osteocytes in cortical bone diaphysis using the SADS assay. Analysis of perigonadal adipose tissue $p 16^{I n k 4 a}$ mRNA expression levels (d), and (e,f) staining for senescence-associated $\beta$-galactosidase- (SA- $\beta$-Gal) positive cells (see arrows [in e], magnification $\times 10$; scale bars, $400 \mu \mathrm{m} ; 100 \mu \mathrm{m}$ in inset in [e]), with (g) quantification of the percentage of SA- $\beta$-Gal-positive cells. (h) Representative $\mu$ CT images $(n=13$ vehicle- 
and $10 \mathrm{D}+\mathrm{Q}$-treated mice) of bone microarchitecture at the lumbar spine of vehicle- versus $\mathrm{D}+\mathrm{Q}-$ treated male C57BL/6 mice. Quantification of $\mu \mathrm{CT}$-derived (i) bone volume fraction (BV/TV; \%), (j) trabecular number (Tb.N; 1/mm), (k) trabecular thickness (Tb.Th; mm), (l) trabecular separation (Tb.Sp; mm), and (m) structure model index (SMI) at the lumbar spine. (n) Representative $\mu \mathrm{CT}$ images ( $n=13$ vehicle- and $10 \mathrm{D}+\mathrm{Q}$-treated mice) of bone microarchitecture at the femur. Quantification of $\mu \mathrm{CT}$-derived (o) cortical thickness $(\mathrm{Ct}$.Th, $\mathrm{mm}$ ) and (p) failure load (N). Histomorphometric quantification at the femoral endocortical surface of (q) osteoclast numbers per bone perimeter (N.Oc/B.Pm;/mm), (r) osteoblast numbers per bone perimeter (N.Ob/B.Pm;/mm), (s) endocortical mineral apposition rate (MAR; $\mathrm{mcm} / \mathrm{d}$ ), and (t) endocortical bone formation rate per bone surface (BFR/BS; $\left.\mathrm{mcm}^{3} / \mathrm{mcm}^{2} / \mathrm{d}\right)(n=8 /$ group$)$. Data represent Mean \pm SEM (error bars). $* P<0.05 ; * * P<$ $0.01 ; * * * P<0.001$ (independent samples $t$-test or Wilcoxon rank-sum test, as appropriate). 

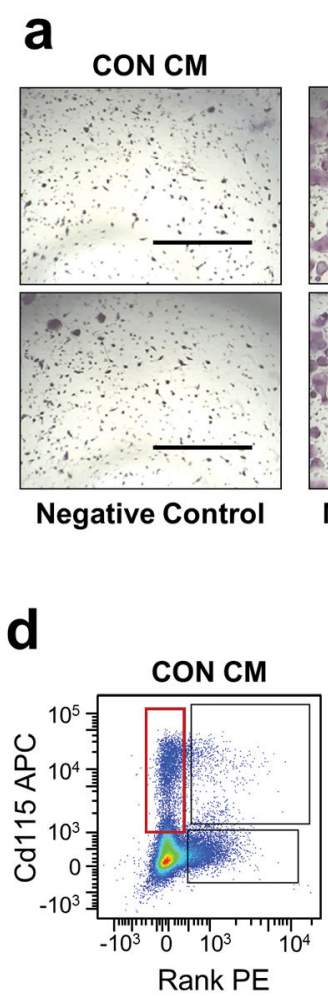

\section{SEN CM}

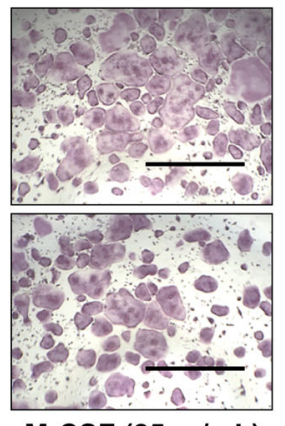

$M-C S F(25 n g / m L)$
b $\odot$ CONCM C
$\triangle$ SEN CM
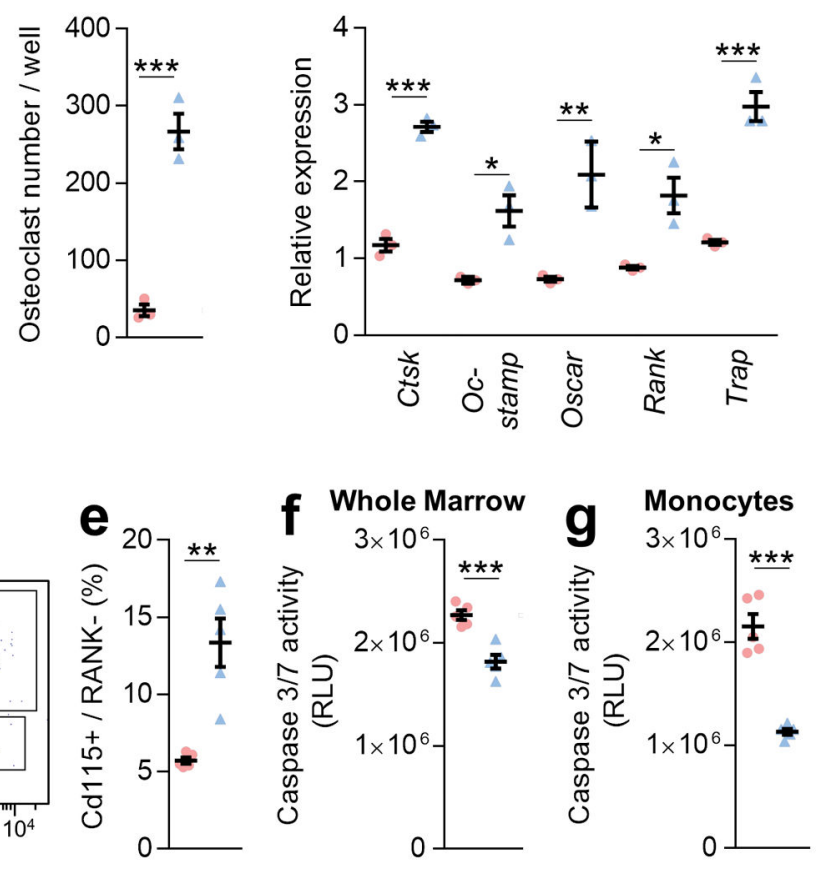
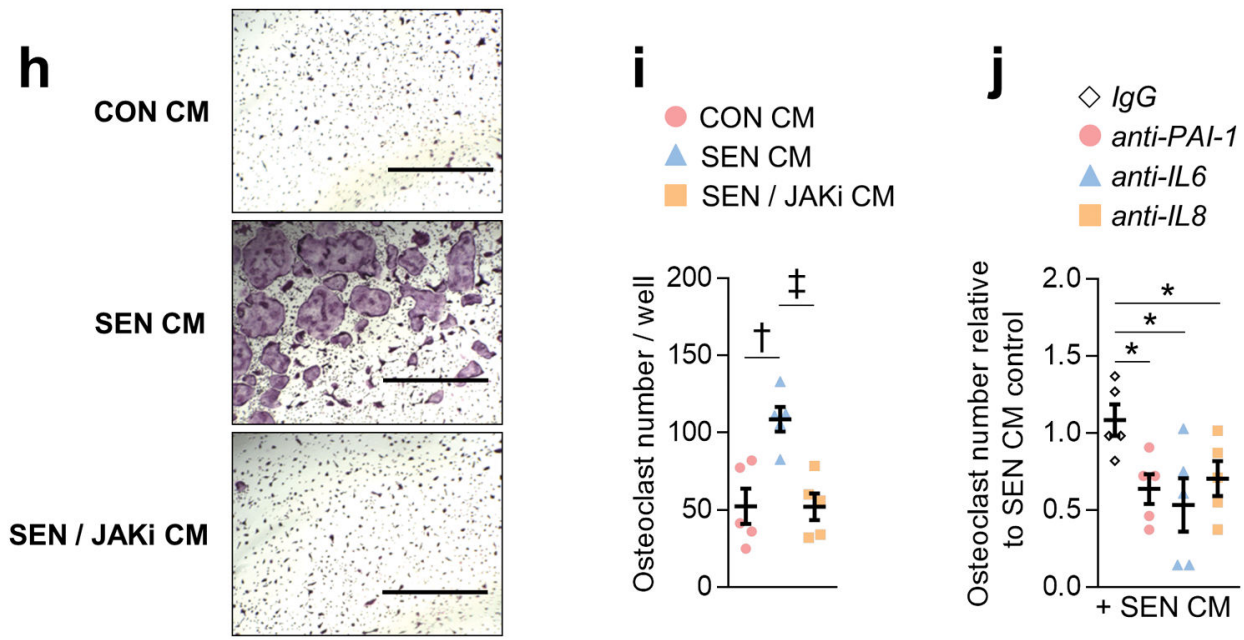

Fig. 3.

The SASP increases osteoclastogenesis in vitro by promoting the survival of monocyte osteoclast progenitors. (a) Representative images (from three individual biological replicates) of tartrate-resistant acid phosphatase (TRAP) stained osteoclast cultures (magnification $\times 4$; scale bars, $1000 \mu \mathrm{m}$ ) and (b) osteoclast numbers per well following four days of osteoclast differentiation of bone marrow cells pre-treated with vehicle (negative control), M-CSF (25ng/mL), control (CON) conditioned medium (CM), or senescent (SEN) $\mathrm{CM}$ ( $n=3$ /group). (c) rt-qPCR analysis of osteoclast genes in bone marrow cells pre-treated with CON CM or SEN CM following two days of osteoclast differentiation; gene expression 
was denoted as fold change relative to vehicle (negative control) pre-treated osteoclast cultures ( $n=3 /$ group). (d, e) Flow cytometric analysis of Cd115+ (monocyte marker) and Rank- (osteoclast progenitor marker) cells in non-adherent population following $24 \mathrm{hrs}$ of treatment with CON CM or SEN CM. (d) Representative images (from five individual biological replicates) of flow cytometric gating with the Cd115+/Rank- monocyte population outlined in red (5.4\% in CON CM and $14.2 \%$ in SEN CM) and (e) average Cd115+/Rank- percentages in CON CM versus SEN CM ( $n=5$ /group; see Supplementary Fig. 15 for flow cytometry gating strategy). (f,g) Apoptosis, as measured by cleavage of a luminogenic caspase-3/7 substrate (RLU), in (f) whole marrow and (g) monocyte-enriched cultures following $24 \mathrm{hrs}$ of treatment with CON CM or SEN CM ( $n=5 /$ group). (h) Representative images (from five individual biological replicates) of TRAP stained osteoclast cultures (magnification $\times 4$; scale bars, $1000 \mu \mathrm{m}$ ) and (i) osteoclast numbers per well following three days of osteoclast differentiation of bone marrow cells pre-treated with

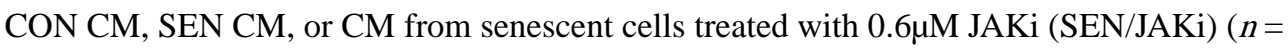
5/group). (j) Osteoclast numbers per well following three days of osteoclast differentiation of bone marrow cells pre-treated with SEN CM in the presence of control $\operatorname{IgG}(20 \mu \mathrm{g} / \mathrm{mL})$, anti-PAI-1 $(5 \mu \mathrm{g} / \mathrm{mL})$, anti-IL-6 $(5 \mu \mathrm{g} / \mathrm{mL})$, or anti-IL-8 $(20 \mu \mathrm{g} / \mathrm{mL})(n=5 /$ group$)$; osteoclast numbers are expressed relative to SEN CM control. Data represent Mean \pm SEM (error bars). $* P<0.05 ; * * P<0.01$; $* * * P<0.001$ (independent samples $t$-test or Wilcoxon ranksum test, as appropriate); ${ }^{\dagger} P<0.01$ (ANOVA versus CON CM followed by Bonferroni posthoc test); ${ }^{\ddagger} P<0.01$ (ANOVA versus SEN CM followed by the Bonferroni post-hoc test). 

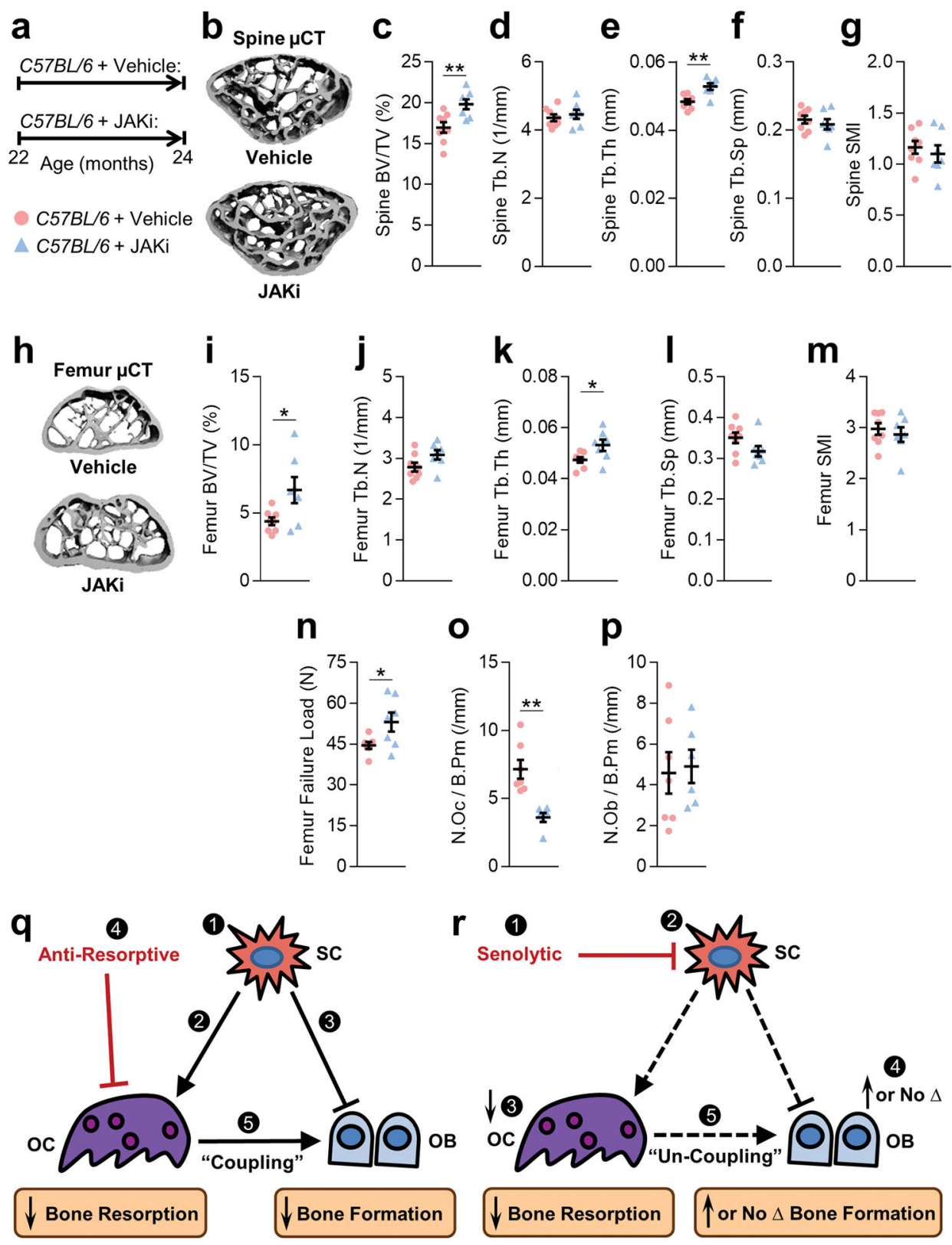

Fig. 4.

Suppression of the SASP by treatment with the JAK1/2 inhibitor, ruxolitinib, prevents agerelated bone loss. (a) Experimental design for testing the effect of SASP inhibition by the JAK1/2 inhibitor, ruxolitinib, on age-related bone loss: 22-month-old male C57BL/6 mice were randomized to either vehicle $(n=8)$ or JAKi $(n=7)$ treatments daily for two months. (b) Representative $\mu \mathrm{CT}$ images of bone microarchitecture at the lumbar spine ( $n=8$ vehicleand 7 JAKi-treated mice). Quantification of $\mu$ CT-derived (c) bone volume fraction (BV/TV; $\%$ ), (d) trabecular number (Tb.N; 1/mm), (e) trabecular thickness (Tb.Th; mm), (f) trabecular separation (Tb.Sp; mm), and (g) structure model index (SMI). (h) Representative $\mu \mathrm{CT}$ images ( $n=8$ vehicle- and $7 \mathrm{JAKi}$-treated mice) of bone microarchitecture at the femoral metaphysis. Quantification of femoral metaphysis $\mu$ CT-derived (i) BV/TV (\%), (j) 
Tb.N (1/mm), (k) Tb.Th (mm), (I) Tb.Sp (mm), (m) structure model index (SMI), and (n) failure load $(\mathrm{N})$. Femoral metaphysis trabecular quantification of (o) osteoclast numbers per bone perimeter (N.Oc/B.Pm; 1/mm)] and (p) osteoblast number per bone perimeter (N.Ob/ B.Pm; $1 / \mathrm{mm}$ )]. Data represent Mean \pm SEM (error bars). $* P<0.05 ; * * P<0.01 ; * * * P<$ 0.001 (independent samples $t$-test or Wilcoxon rank-sum test, as appropriate). (q,r) Comparing the effects of anti-resorptive versus senolytic therapies on bone metabolism. (q) (1) Senescent cells (SCs) accumulate in the bone microenvironment with aging where they (2) increase bone resorption by osteoclasts (OCs) and (3) decrease bone formation by osteoblasts (OBs). (4) Anti-resorptive therapy inhibits/eliminates OCs and decreases bone resorption; due to (5) coupling there is a concomitant reduction in bone formation. (r) (1) Senolytic therapy reduces the burden of (2) SCs, which in turn (3) suppresses bone resorption with (4) either an increase (cortical bone) or maintenance (trabecular bone) in bone formation leading to (5) un-coupling between OCs and OBs. 